\title{
Therapeutic effect of hydrogen injected subcutaneously on onion poisoned dogs
}

\author{
Jinghua Zhao ${ }^{1}$, Ming Zhang ${ }^{1}$, Yue $\mathrm{Li}^{1}$, \\ Zhiheng Zhang ${ }^{1}$, Mingzi Chen ${ }^{1}$, Tao Liu ${ }^{1}$, \\ Jiantao Zhang ${ }^{1}$, Anshan Shan ${ }^{2}$ \\ ${ }^{1}$ Heilongjiang Key Laboratory for Laboratory Animals and Comparative Medicine, College of Veterinary Medicine, \\ ${ }^{2}$ College of Animal Science and Technology, Northeast Agriculture University, Harbin 150030, China \\ zhangjiantao@neau.edu.cn; shanasvip@163.com
}

Received: July 11, 2017 Accepted: December 6, 2017

\begin{abstract}
Introduction: The purpose of this study was to investigate the therapeutic effect of hydrogen on the therapy of onion poisoned dogs. Material and Methods: A total of 16 adult beagle dogs were divided into two groups (control and hydrogen) and all were fed dehydrated onion powder at the dose of $10 \mathrm{~g} / \mathrm{kg}$ for three days. The dogs of the experimental group were given subcutaneous injection of $0.2 \mathrm{~mL} / \mathrm{kg}$ of hydrogen for 12 days after making the poisoned model successful. Blood samples were collected before feeding onions, one day before injecting hydrogen, and $2 \mathrm{~h}$ after the injection of hydrogen on days 1, 3, 5, 7, 9, and 12. Control dogs were not treated with hydrogen. Results: The levels of leukocyte production, anaemia, red blood cell degeneration which was reflected by the values of Heinz body count, haemolytic ratio, and oxidative products in hydrogen treated group were lower than in control dogs on some days. The capacity of medullary haematopoiesis that was based on reticulocyte counts, and the antioxidation in hydrogen group were higher compared with control group. However, the differences in renal function were not obvious in both groups. Conclusion: Accordingly, it was concluded that subcutaneous injection of hydrogen could alleviate the symptoms in onion poisoned dogs.
\end{abstract}

Keywords: dogs, onion poisoning, hydrogen, therapy.

\section{Introduction}

The onions have been widely recognised to possess some medicinal properties, such as promoting blood circulation, anti-bacterial and anti-inflammation properties, aside from their nutritional value and taste benefits for humans $(6,26)$. However, some animal species may show symptoms of poisoning after eating onions. Among animal species, cats and dogs are relatively susceptible to onion-induced oxidative damage, followed by cattle, horses, sheep, goats, rats, and mice, in order of increasing resistance $(8,21)$.

The toxic elements in onions are mainly n-propyl disulfide $(5,22)$ and sodium n-propyl thiosulfate $(18$, 25), which can increase the counts of Heinz bodies and decrease glutathione concentration in erythrocytes. The mechanism that causes onion poisoning in $\operatorname{dogs}$ is attributed to damage of the antioxidant system in erythrocytes, causing series of pathological changes in erythrocyte membrane and intracellular materials, and finally leading to haemolytic anaemia (24). Clinical symptoms mainly include red urine, a decrease in red blood cells, as well as a substantial increase in Heinz bodies and reticulocyte number. It should be noted that if the severely poisoned dogs are not treated appropriately, they will die eventually. It is reported that raw onions, cooked onions, dehydrated onions, and crushed onions are all potentially toxic for dogs (7).

Hydrogen is the most abundant element in the universe. Until recently the knowledge on potential biological application of hydrogen was scarce. In 2007, Ohsawa et al. (20) reported that $2 \%$ inhalation of hydrogen gas could ameliorate significantly the cerebral ischaemia reperfusion injury in rats, and it was based on a selective decrease in hydroxyl free radical $(. \mathrm{OH})$ and peroxynitrite $\left(\mathrm{ONOO}^{-}\right)$, which were much more reactive than other reactive oxygen species (ROS) (20). The study caused quickly a widespread concern and scientists had made great progress in medical application of hydrogen molecule. Many published papers have 
indicated that hydrogen has numerous biological effects, such as selective antioxidation (20), anti-infection (10), anti-tumour (17), and anti-radiation (14), and it does not exert any cytotoxicity even at high concentration $(1,13)$. Compared with other known antioxidants, hydrogen has many distinct advantages, such as selective oxidation, non-toxicity, high permeability, no residues, relative safety, and low cost (9).

Combining the main mechanism of the dogs' onion poisoning with biological applications of hydrogen, the purpose of this study was to assess the therapeutic effect of hydrogen on the damages induced by onion poisoning in dogs.

\section{Material and Methods}

Model establishment and sample collection. A total of 16 adult beagle dogs were used in this experiment. The dogs were vaccinated, individually housed, and no substances that would affect the results of the experiment were administered during the experiment. All dogs were divided into two equal groups: control group (group C) in which the dogs did not undergo any treatment after poisoning, and hydrogen group (group $\mathrm{H}_{2}$ ) in which the dogs were injected with hydrogen after poisoning. All dogs were fed dehydrated onion powder at the dose of $10 \mathrm{~g} / \mathrm{kg}$ for three days. Onion powder was mixed with cooked meat when fed. Heinz's ratio of more than $50 \%$ and haematuria indicated that the poisoning model had been successfully established. Hydrogen was produced by hydrogen generator (Saikesaisi, China) and was stored in a sealed bag, using a syringe to extract hydrogen gas from the exhaust hole when needed. The dogs in group $\mathrm{H}_{2}$ were injected hydrogen subcutaneously in the dose of $0.2 \mathrm{~mL} / \mathrm{kg}$ (23) for 12 days, in which the Heinz bodies almost disappeared. Blood samples of two groups were collected from the jugular vein one week before feeding the onion powder (day -7), one day before injecting hydrogen (day 0 ), and $2 \mathrm{~h}$ after injection of hydrogen gas on days $1,3,5,7,9$, and 12 of experimental treatment.

Index measurement and method. Indexes measured included five aspects: clinical symptoms, routine blood indexes, blood smears, liver and kidney function, and antioxidant system of erythrocytes.

In the entire course of the experiment, the clinical symptoms were closely observed, mainly including the following aspects: urine colour, dietary status, body temperature, and status of visible mucosa.

The haematology index was tested timely. Red blood cell (RBC) and white blood cell (WBC) counts, haemoglobin (HGB), and haematocrit (HCT) were determined with a fully automated haematology analyser (MEK-7222K, Japan).

Blood smears were mainly used to detect Heinz bodies and reticulocytes. Fresh blood was stained with Wright Giemsa staining method to count Heinz bodies, which were determined by the percentage of cells that had Heinz bodies within a population of 500 erythrocytes counted (7). Reticulocytes were observed after staining with brilliant cresyl blue. The stain and blood were mixed in a 1:1 ratio, incubated for $15 \mathrm{~min}$ or longer at room temperature, then blood smears were assessed according to conventional criteria. At least 1,000 RBCs were counted and Miller's method was used to make reticulocyte counting precise. The reticulocyte cytoplasm contained light or dark blue mesh structure after staining.

The anticoagulated blood was centrifuged at $2,500 \mathrm{~g} / \mathrm{min}$ for $10 \mathrm{~min}$, and the plasma was used to determine the concentration of biochemical markers characteristic for hepatic and renal functions, and haemolytic ratio. Blood urea nitrogen (BUN), creatinine (CRE), alanine aminotransferase (ALT), aspartate aminotransferase (AST), total bilirubin (TBIL), and direct bilirubin (DBIL) were determined. These compounds were tested using an automated biochemical analyser (IDEXX, USA).

After centrifuging the anticoagulated blood, the precipitated RBCs were washed first and physiological saline was added in a volume three times bigger than that of RBCs, then the solution was centrifuged for $5 \mathrm{~min}$, and the RBCs pellet was washed again in physiological saline. The process was repeated three times until the supernatant was colourless. RBCs were extracted quantitatively and cold distilled water was added proportionately for hemolysate to measure malondialdehyde (MDA), the total antioxidant capacity (T-AOC), reduced glutathione (GSH), catalase (CAT), and the hydroxy radical inhibition activity in the antioxidant system of erythrocytes using reagent kits (Nanjing Jiancheng Bioengineering Institute, China).

Statistical methods. Using SPSS software package (Version 22.0) for statistical data analysis, the results were shown as means \pm standard deviation ( $\pm \mathrm{SD}$ ). Independent-sample $t$-test was adopted to compare results of two groups (group $\mathrm{C}$ and group $\mathrm{H}_{2}$ ) after the test of normality and the assumption for homogeneity of variances. $P$ value $<0.05$ was considered statistically significant.

\section{Results}

Clinical symptoms. All dogs were healthy and active before feeding onions. After feeding onions for three days, all dogs in groups $\mathrm{C}$ and $\mathrm{H}_{2}$ showed haematuria, increased body temperature, and pale visual mucosa. On the $3^{\text {rd }}$ day after the injection of hydrogen, the urine of five dogs in group $\mathrm{H}_{2}$ turned yellow, but only two dogs in group $\mathrm{C}$ showed signs of improvement. On the $10^{\text {th }}$ day after injection of hydrogen, the clinical symptoms of both groups returned to normal.

Routine blood indexes. WBC increased dramatically after poisoning, and both groups reached the peak on day 3 . The counts of WBC in group $\mathrm{H}_{2}$ were lower than those in group C. Significant differences were 
noted on days 7 and $12(\mathrm{P}<0.01)$ (Fig. 1A). RBCs in the two groups decreased after poisoning and reached minimum on day 3 , then began to recover. The counts of $\mathrm{RBC}$ in group $\mathrm{H}_{2}$ were higher than those in group $\mathrm{C}$ on days 5, 9, $12(\mathrm{P}<0.05)$, and on day $7(\mathrm{P}<0.01)$ (Fig. 1B). HGB declined sharply after poisoning and did not recover to normal levels on day 12 . HGB in group $\mathrm{H}_{2}$ was higher than in group $\mathrm{C}$ in the period of hydrogen injection, and the difference was significant on days 7 and $12(\mathrm{P}<0.05)$, and highly significant on day 9 $(\mathrm{P}<0.01)$ (Fig. 1C). HCT decreased until day 3 , then increased slowly after poisoning, and in group $\mathrm{H}_{2}$ was higher than in group $\mathrm{C}$, particularly on day $5(\mathrm{P}<0.01)$ and on day $7(\mathrm{P}<0.05)$ (Fig. 1D).

Blood smear examinations and haemolytic ratio. Heinz bodies in group $\mathrm{H}_{2}$ disappeared completely first and were lower than in group $\mathrm{C}$, and a significant difference was observed on days 5, $7(\mathrm{P}<0.05)$, and 9 $(\mathrm{P}<0.01)$ (Fig. 2A). Reticulocyte counts in group $\mathrm{H}_{2}$ were higher than in group $\mathrm{C}$, especially on days 3 $(\mathrm{P}<0.05), 5$, and $7(\mathrm{P}<0.01)$ (Fig. 2B). The haemolytic ratio counts increased first and then decreased in the two groups, and both reached the peak on day 3 . The counts in group $\mathrm{H}_{2}$ were lower than in group $\mathrm{C}$, particularly on days 3,9 , and $12(\mathrm{P}<0.01)$ (Fig. $2 \mathrm{C})$.

Liver and renal function measurements. The ALT in group $\mathrm{H}_{2}$ was lower than in group $\mathrm{C}$ during hydrogen injection and showed significant difference on day $7(\mathrm{P}<0.01)$. The AST in group $\mathrm{H}_{2}$ was lower than in group $\mathrm{C}$ on days $1,3,5$, and 7 and there was a significant difference on day $5(\mathrm{P}<0.01)$. The values of TBIL and DBIL had similar trends and TBIL values in group $\mathrm{H}_{2}$ were lower than in group $C$ (except on days 1 and 12), particularly on day $5(\mathrm{P}<0.01)$. The changing trends of BUN and CRE were irregular with fluctuations in the normal range. There was no significant difference between the two groups at other time points except on day 9 for CRE $(\mathrm{P}<0.05)$ (Fig. $3 \mathrm{~A}-\mathrm{F})$.

Antioxidant system of erythrocytes. The MDA level in group $\mathrm{H}_{2}$ were lower than in group $\mathrm{C}$ (except day 1) and significant difference was noted on days $9(\mathrm{P}<0.05)$, 5 , and $7(\mathrm{P}<0.01)$. The changing trend for T-AOC after poisoning was irregular. T-AOC values in group $\mathrm{H}_{2}$ were higher than in group $\mathrm{C}$ (except day 1 ), especially on days $3,9(\mathrm{P}<0.01)$, and $12(\mathrm{P}<0.05)$. The hydroxy radical inhibition activity declined sharply after poisoning, and the values in group $\mathrm{H}_{2}$ were higher than in group $\mathrm{C}$ from the third day after injecting hydrogen, with significant difference observed on day $7(\mathrm{P}<0.05)$ and on days 3 and $9(\mathrm{P}<0.01)$. The values of CAT did not have a regular changing trend and there was no significant difference between the two groups. The concentration of GSH in the two groups decreased rapidly after poisoning and reached the minimum on day 3 ; then it began to recover. The values in group $\mathrm{H}_{2}$ reached the maximum on day 7 , then decreased and fluctuated as the values before poisoning. The values in group $\mathrm{H}_{2}$ were higher than in group $\mathrm{C}$ on days 5 and $7(\mathrm{P}<0.01)$. Detailed results are shown in Table 1.
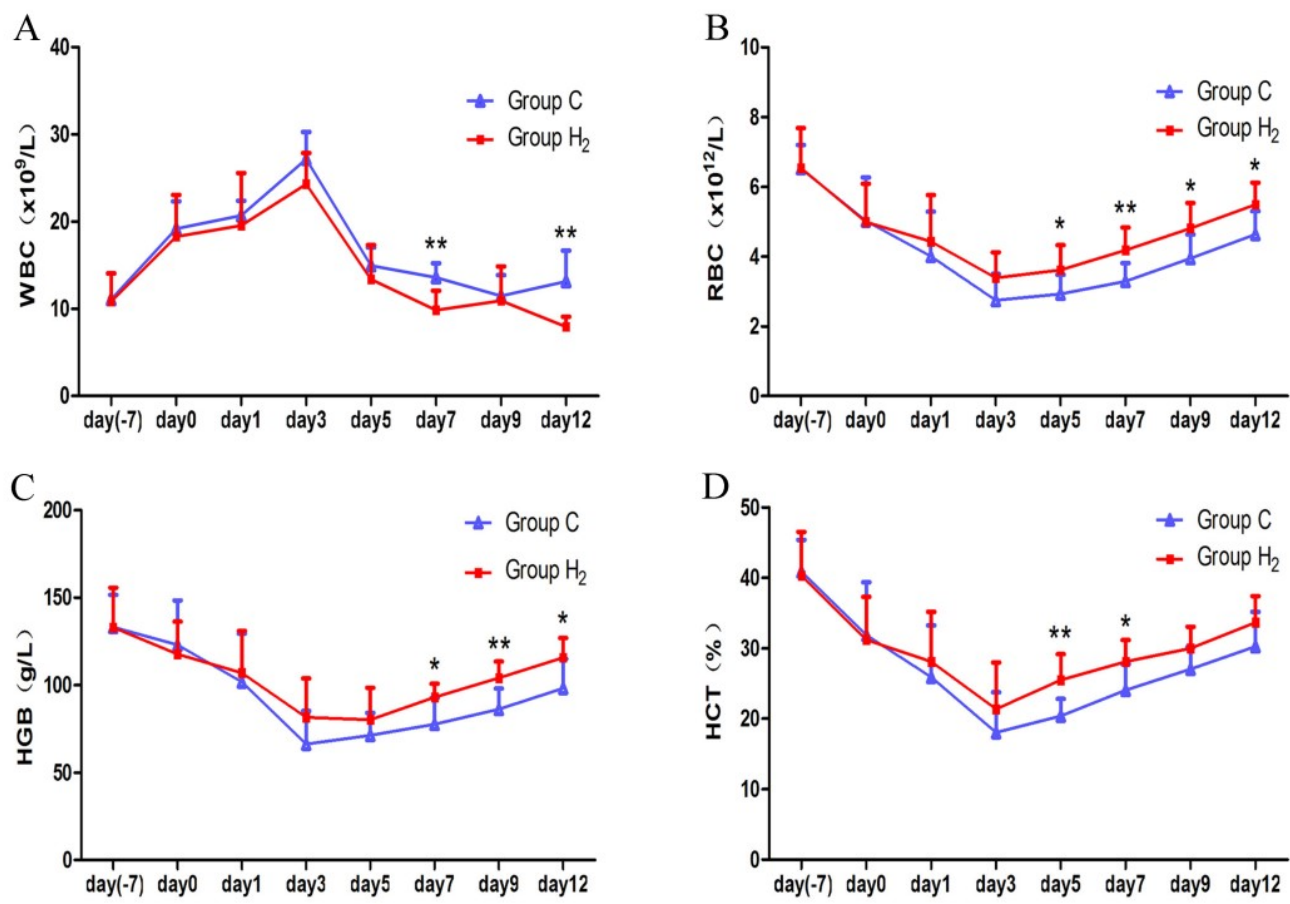

Fig. 1. Comparison of the values of white blood cells (WBCs), red blood cells (RBCs), haemoglobin (HGB), and haematocrit (HCT) between the control group (group $\mathrm{C}$ ) and hydrogen group (group $\mathrm{H}_{2}$ ) on day 7 before injecting hydrogen, on day 0 (one day before injecting hydrogen), and on days $1,3,5,7,9$, and 12 during the injection of hydrogen. $* \mathrm{P}<0.05, * * \mathrm{P}<0.01$ 

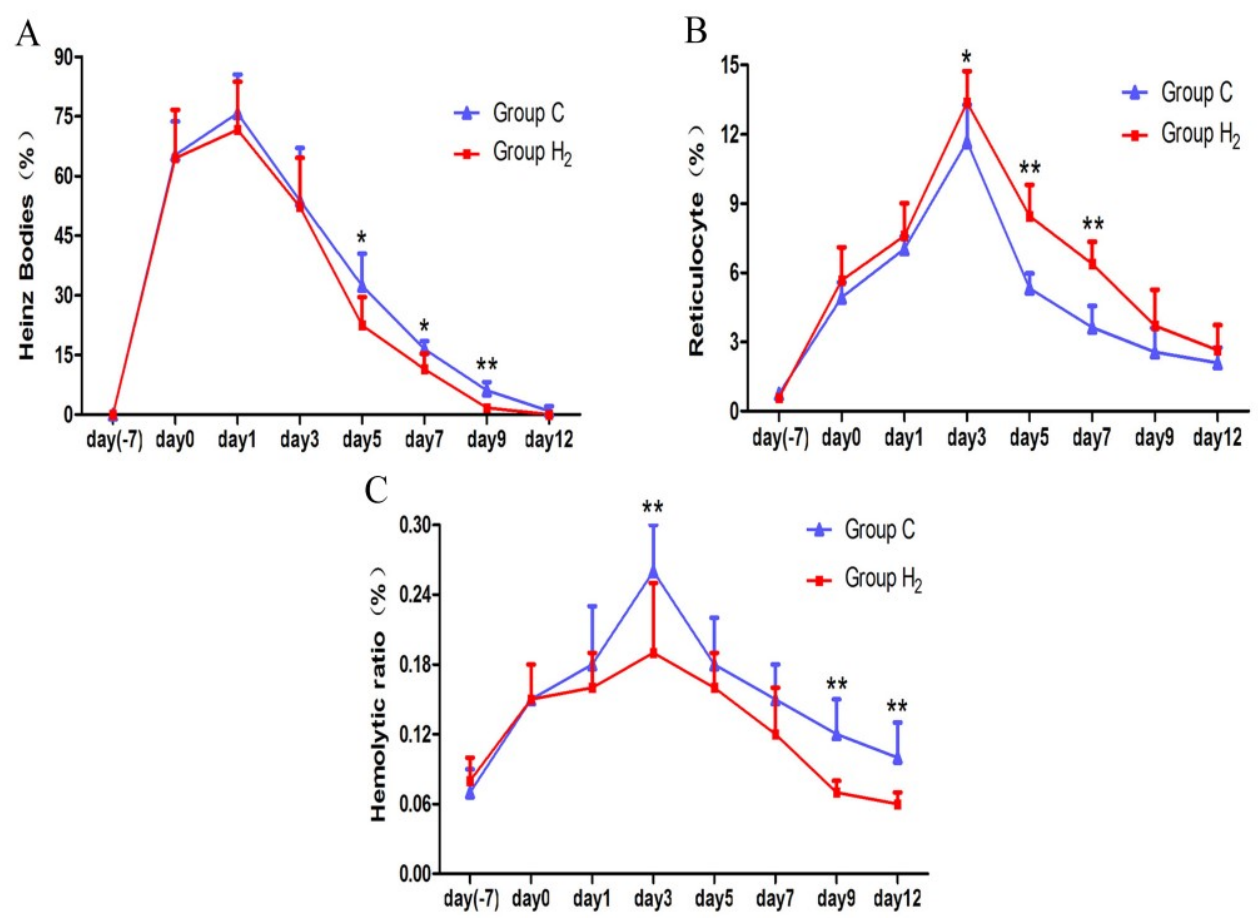

Fig. 2. Comparison of counts of Heinz bodies, reticulocytes, and haemolytic ratio between the control group (group C) and hydrogen group (group $\mathrm{H}_{2}$ ) on day 7 before injecting hydrogen, day 0 (one day before injecting hydrogen), and on days $1,3,5,7,9$, and 12 during the injection of hydrogen. $* \mathrm{P}<0.05, * * \mathrm{P}<0.01$

Table 1. The erythrocyte antioxidant system results (mean $\pm \mathrm{SD}$ ) in the control group (group C) and hydrogen group (group $\mathrm{H}_{2}$ ) on day 7 before injecting hydrogen, on day 0 (one day before injecting hydrogen), and on days $1,3,5,7,9$, and 12 during the injection of hydrogen gas

\begin{tabular}{lllllll}
\hline Time & Group & MDA $(\mathrm{nmol} / \mathrm{mL})$ & T-AOC $(\mathrm{U} / \mathrm{mL} \mathrm{RBC})$ & $\cdot \mathrm{OH}$ IA $(\mathrm{U} / \mathrm{mgHb})$ & $\mathrm{CAT}(\mathrm{U} / \mathrm{mgHb})$ & $\mathrm{GSH}(\mu \mathrm{mol} / \mathrm{L})$ \\
\hline \multirow{2}{*}{ Day (-7) } & Group C & $4.5 \pm 0.98$ & $324.63 \pm 28.03$ & $89.73 \pm 8.35$ & $5.05 \pm 1.15$ & $166.09 \pm 16.46$ \\
& Group $\mathrm{H}_{2}$ & $4.27 \pm 0.92$ & $318.08 \pm 21.87$ & $84.12 \pm 8.89$ & $4.81 \pm 1.11$ & $165.56 \pm 12.95$ \\
\multirow{2}{*}{ Day 0 } & Group C & $9.48 \pm 1.27$ & $279.95 \pm 25.55$ & $55.91 \pm 11.68$ & $2.88 \pm 0.54$ & $133.63 \pm 9.63$ \\
& Group $\mathrm{H}_{2}$ & $8.69 \pm 0.98$ & $281.2 \pm 22.81$ & $53.40 \pm 11.96$ & $2.83 \pm 0.48$ & $125.68 \pm 9.11$ \\
Day 1 & Group C & $16.79 \pm 1.67$ & $232.12 \pm 20.52$ & $38.06 \pm 6.79$ & $4.04 \pm 1.22$ & $93.44 \pm 13.77$ \\
& Group $\mathrm{H}_{2}$ & $17.41 \pm 0.7$ & $231.21 \pm 27.33$ & $34.8 \pm 5.9$ & $3.89 \pm 0.81$ & $78.59 \pm 11.37^{*}$ \\
Day 3 & Group C & $16.48 \pm 2.16$ & $240.47 \pm 21.2$ & $22.36 \pm 3.66$ & $3.64 \pm 1.23$ & $50.35 \pm 8.42$ \\
& Group $\mathrm{H}_{2}$ & $16.0 \pm 2.87$ & $299.08 \pm 12.82^{* *}$ & $27.28 \pm 2.56^{* *}$ & $4.68 \pm 1.06$ & $56.35 \pm 6.0$ \\
Day 5 & Group C & $9.6 \pm 1.66$ & $256.98 \pm 26.59$ & $53.66 \pm 6.57$ & $5.38 \pm 1.16$ & $104.53 \pm 8.08$ \\
& Group $\mathrm{H}_{2}$ & $7.19 \pm 1.34^{* *}$ & $274.56 \pm 31.59$ & $55.82 \pm 8.38$ & $6.37 \pm 1.1$ & $122.68 \pm 6.77^{* *}$ \\
Day 7 & Group C & $6.94 \pm 1.2$ & $304.76 \pm 35.84$ & $65.43 \pm 8.38$ & $6.34 \pm 1.62$ & $143.91 \pm 9.86$ \\
& Group $\mathrm{H}_{2}$ & $5.03 \pm 1.18^{* *}$ & $313.13 \pm 24.93$ & $73.87 \pm 6.98^{*}$ & $5.69 \pm 1.36$ & $201.07 \pm 14.3 * *$ \\
Day 9 & Group C & $4.54 \pm 0.57$ & $247.79 \pm 24.56$ & $71.1 \pm 6.98$ & $6.45 \pm 1.61$ & $171.8 \pm 12.01$ \\
& Group $\mathrm{H}_{2}$ & $3.73 \pm 0.86^{*}$ & $345.56 \pm 37.97^{* *}$ & $82.3 \pm 6.46^{* *}$ & $7.14 \pm 0.91$ & $164.64 \pm 12.84$ \\
Day 12 & Group C & $2.77 \pm 0.42$ & $270.88 \pm 22.56$ & $79.81 \pm 11.41$ & $7.27 \pm 1.71$ & $184.3 \pm 20.66$ \\
& Group $\mathrm{H}_{2}$ & $2.47 \pm 0.15$ & $304.01 \pm 30.42^{*}$ & $77.07 \pm 12.7$ & $7.76 \pm 0.71$ & $170.37 \pm 7.31$ \\
\hline
\end{tabular}

MDA - malondialdehyde; T-AOC - total antioxidant capacity; $\cdot$ OH IA - hydroxy radical inhibition activity; CAT - catalase; GSH - reduced glutathione. $* \mathrm{P}<0.05, * * \mathrm{P}<0.01$ 

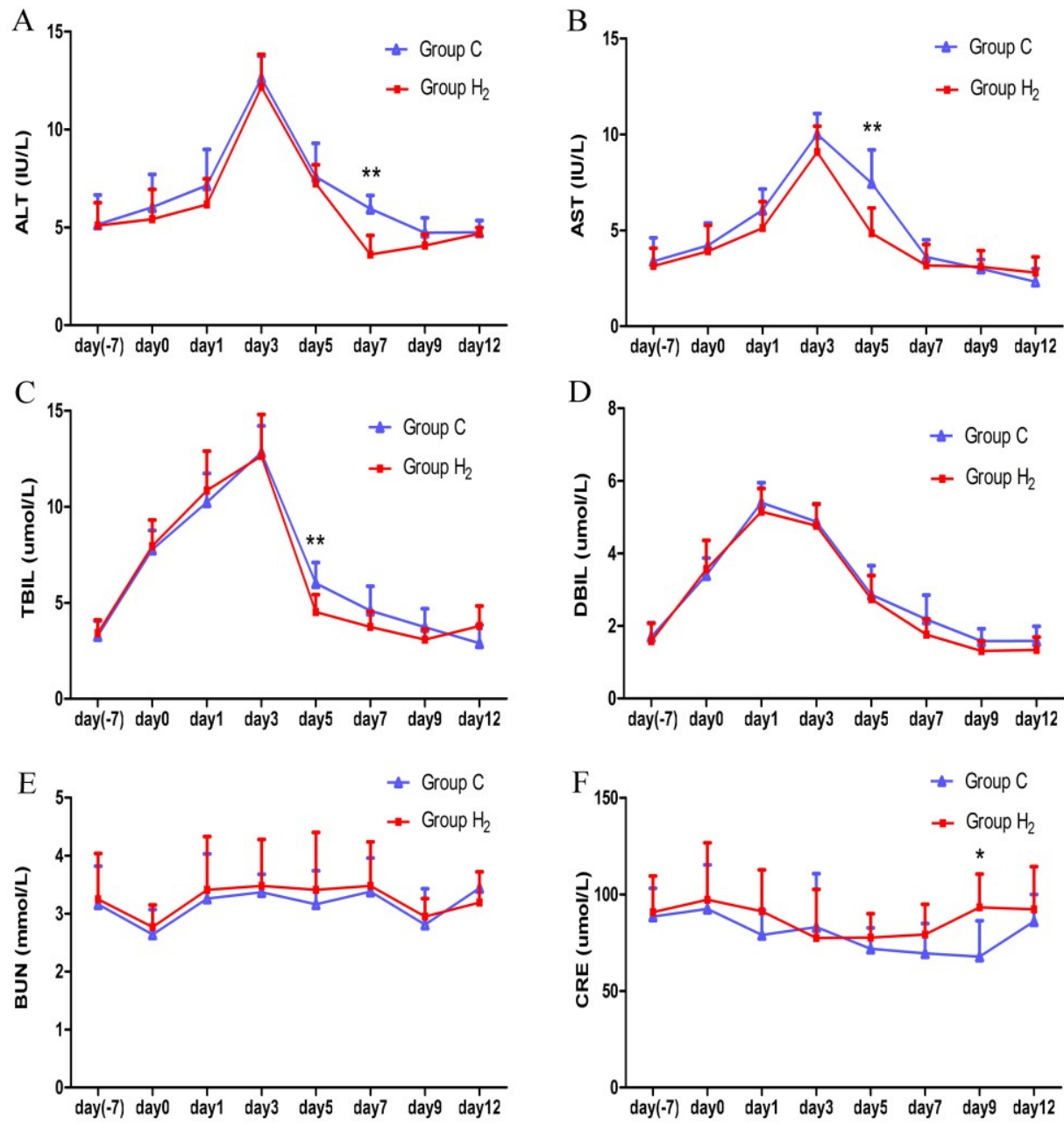

Fig. 3. Comparison of the values of alanine aminotransferase (ALT), aspartate aminotransferase (AST), total bilirubin (TBIL), direct bilirubin (DBIL), blood urea nitrogen (BUN), and creatinine (CRE) between the control group (group $\mathrm{C}$ ) and hydrogen group (group $\mathrm{H}_{2}$ ) on day 7 before injecting hydrogen, on day 0 (one day before injecting hydrogen), and on days $1,3,5,7,9$ and 12 during the injection of hydrogen. $* \mathrm{P}<0.05, * * \mathrm{P}<0.01$

\section{Discussion}

In this study, the model of onion poisoning in dogs was successfully established, and typical haemolytic anaemia symptoms were observed in the animals. A previous study (11) has revealed that onion poisoning occurs in dogs when the amount of onions fed accounts for more than $0.5 \%$ of the animal's weight. Considering the different moisture content of fresh onions, dehydrated onion powder was used in this study because of ease of administration and better control of the dose (7). The final dose of onion powder was based on the results of a preliminary study.

The routine blood indexes, blood smear, biochemical markers of liver and kidney function, and erythrocyte antioxidant system all basically followed the trend of poisoning-recovering. Heinz bodies and MDA increased to peak and then decreased, which was consistent with the results of previous research (24). Some physiological indicators suggested that the dogs in group $\mathrm{H}_{2}$ recovered first in relation to group $\mathrm{C}$, e.g.
Heinz bodies which disappeared first in the group $\mathrm{H}_{2}$. However, all dogs in two groups returned to healthy physiological state by the end of experiment. The dogs in group $\mathrm{C}$ were also self-healing finally due to the effective removal system of RBC, which included two sets of antioxidant systems. One was enzymatic system, such as superoxide dismutase (SOD), and the other was non-enzymatic system including vitamin $\mathrm{C}$, glutathione, vitamin E, etc. $(4,16)$. These specific scavenging systems weaken the toxic effects of hydrogen peroxide and other ROS (2), and eventually relieved the symptoms of poisoning in the dogs which recovered in the end.

There was no drastic change in CRE and BUN in comparison to the period before feeding onions. It was because the kidneys had a strong reserve and compensatory capacity, so CRE and BUN were usually at the healthy level in the early detection of renal damage, until substantial impairment of the kidneys was observed or the development of end-stage renal disease occurred. The results indicated that the 
poisoned dogs did not show severe kidney damage in the experimental process, which corresponded to the previous study stating that serum BUN or UC ratio and glomerular filtration rates (GFR) did not show an increase in anaemic dogs (15).

Hydrogen influenced the selective oxidation and could react with the active oxygen, such as $\cdot \mathrm{OH}$ and $\mathrm{ONOO}^{-}$, that had very strong oxidation directly (20). The results obtained demonstrated that the levels of hydroxy radical inhibition activity in group $\mathrm{H}_{2}$ were significantly higher than in group $\mathrm{C}$ from the third day of hydrogen injection, and dogs of the group $\mathrm{H}_{2}$ recovered first. T-AOC, GSH, CAT, and MDA in group $\mathrm{H}_{2}$ showed significant differences on some days after hydrogen injection, suggesting that the hydrogen could improve the antioxidant levels of the body and lower the levels of oxidative products, which was also shown in previous studies $(3,27)$. This was not proven to be the direct effect of hydrogen, but it might be related to the mutual relation between the oxidation system, as high level of intracellular GSH could cause reduction reaction with $\mathrm{H}_{2} \mathrm{O}_{2}$, which had a direct correlation with CAT.

The counts of reticulocytes in group $\mathrm{H}_{2}$ were significantly higher than in group $\mathrm{C}$, indicating that hydrogen could improve bone marrow haematopoietic capacity (12), and the trend was consistent with the changes of RBC. MDA was one of the lipid peroxidation products. Hydrogen peroxide and MDA could oxidise the sulfhydryl groups of haemoglobin, causing the denaturation of haemoglobin and the formation of Heinz bodies $(19,24)$. The number of Heinz bodies between the two groups was in conformity with MDA change.

The lipid peroxidation also decreased the fluidity and deformation of erythrocyte membrane (24), resulting in more serious damage of erythrocytes, which would cause haemoglobinaemia and haemoglobinuria. The values of the haemolytic rate, which had positive correlation with the injury degree of erythrocyte membrane, were lower in group $\mathrm{H}_{2}$ than in group $\mathrm{C}$ on some days, showing that hydrogen could inhibit the lipid peroxidation at a certain extent, reduce the oxidative damage of erythrocyte membrane, leading to reduction of the haemolysis degree in the poisoned dogs. The significant differences in the blood indexes observed in the present study between the two groups indicate that hydrogen could reduce the oxidative damage of the erythrocytes, and then lead to reduction in the degree of haemolytic anaemia.

In conclusion, the results indicate that continuous injection of hydrogen in onion poisoned dogs would relieve haemolytic anaemia and increase bone marrow haematopoietic ability. Hydrogen could also reduce the oxidative damage of erythrocyte membrane by reducing oxidation products and increasing antioxidant substances, thus causing reduction of the number of Heinz bodies and haemolysis rate. Hydrogen could be considered as an auxiliary treatment drug for onion poisoned dogs in the future.

Conflict of Interests Statement: The authors declare that there is no conflict of interests regarding the publication of this article.

Financial Disclosure Statement: This study was funded by the National Natural Science Foundation of China, China Postdoctoral Science Foundation, and Young Talents Project of Northeast Agricultural University.

Animal Rights Statement: All animal procedures were approved by the Laboratory Animal Care and Use Committee of Northeast Agricultural University.

\section{References}

1. Abraini J.H., Gardette-Chauffour M.C., Martinez E., Rostain J.C., Lemaire C.: Psychophysiological reactions in humans during an open sea dive to $500 \mathrm{~m}$ with a hydrogenhelium-oxygen mixture. J Appl Physiol 1994, 76, 1113-1118.

2. Aoki T., Nishimura M., Kataoka H., Ishibashi R., Nozaki K., Hashimoto N.: Reactive oxygen species modulate growth of cerebral aneurysms: a study using the free radical scavenger edaravone and p47phox ${ }^{-/-}$mice. Lab Invest 2009, 89, 730-741.

3. Cai W.W., Zhang M.H., Yu Y.S., Cai J.H.: Treatment with hydrogen molecule alleviates $\mathrm{TNF} \alpha$-induced cell injury in osteoblast. Mol Cell Biochem 2013, 373, 1-9.

4. Chow C.K.: Vitamin E and oxidative stress. Free Radical Bio Med 1991, 11, 215-232.

5. Cope R.B.: Allium species poisoning in dogs and cats. Vet Med 2005, 100, 562-566.

6. Furusawa M., Tsuchiya H., Nagayama M., Tanaka T., Oyama M., Ito T., Iinuma M., Takeuchi H.: Cell growth inhibition by membrane-active components in brownish scale of onion. J Health Sci 2006, 52, 578-584.

7. Harvey J.W., Rackear D.: Experimental onion-induced hemolytic anemia in dogs. Vet Pathol 1985, 22, 387-392.

8. Heidarpour M., Fakhrieh M., Aslani M.R., Mohri M., Keywanloo M.: Oxidative effects of long-term onion (Allium cepa) feeding on goat erythrocytes. Comp Clin Pathol 2013, 22, 195-202.

9. Huang C.S., Kawamura T., Toyoda Y., Nakao A.: Recent advances in hydrogen research as a therapeutic medical gas. Free Radical Res 2010, 44, 971-982.

10. Kajiya M., Sato K., Silva M.J., Ouhara K., Do P.M., Shanmugam K.T.: Hydrogen from intestinal bacteria is protective for concanavalin A-induced hepatitis. Biochem Biophys Res Commun 2009, 386, 316-321.

11. Kay J.M.: Onion toxicity in a dog. Mod Vet Pract 1983, 64, $477-478$.

12. Lesesve J.F., Lacombe F., Marit G., Bernard P., Belloc F., Reiffers J.: High fluorescence reticulocytes are an indicator of bone marrow recovery after chemotherapy. Eur J Haematol 1995, 54, 61-63.

13. Lillo R.S., Parker E.C.: Mixed-gas model for predicting decompression sickness in rats. J Appl Physiol 2000, 89, 2107-2116.

14. Liu C., Cui J., Sun Q., Cai J.: Hydrogen therapy may be an effective and specific novel treatment for acute radiation syndrome. Med Hypotheses 2010, 74, 145-146.

15. Lobetti R.: Changes in the serum urea: Creatinine ratio in dogs with babesiosis, haemolytic anaemia, and experimental haemoglobinaemia. Vet J 2011, 19, 253-256. 
16. Masotti L., Casali E., Galeoti T.: Lipid peroxidation in tumor cells. Free Radic Biol Med 1988, 4, 377-386.

17. Nakashima-Kamimura N., Mori T., Ohsawa I., Asoh S., Ohta S.: Molecular hydrogen alleviates nephrotoxicity induced by an anticancer drug cisplatin without compromising anti-tumor activity in mice. Cancer Chemother Pharm 2009, 64, 753-761.

18. Ogawa E., Akahori F., Kobayashi K.: In vitro studies on the breakdown of canine erythrocytes exposed to the onion extract. J Vet Med Sci 1985, 47, 719-729.

19. Ogawa E., Kawakami A., Yagi T., Amaya T., Fujise H., Takahashi R.: Oxidative damage to the membrane of canine erythrocytes with inherited high Na, K-ATPase activity. J Vet Med Sci 1992, 54, 57-62.

20. Ohsawa I., Ishikawa M., Takahashi K., Watanabe M., Nishimaki K., Yamagata K., Katsura K., Katayama Y., Asoh S., Ohta S.: Hydrogen acts as a therapeutic antioxidant by selectively reducing cytotoxic oxygen radicals. Natl Med 2007, 13, 688-694.

21. Rae H.A.: Onion toxicosis in a herd of beef cows. Can Vet J $1999,40,55-57$
22. Simmons D.M.: Onion breath. Vet Tech 2001, 22, 424-427.

23. Song C., Wei J.: Effect of hydrogen injected subcutaneously on testicular tissues of rats exposed to cigarette smoke. Int J Clin Exp Med 2015, 8, 5565-5570.

24. Tang X., Xia Z., Yu J.: An experimental study of hemolysis induced by onion (Allium cepa) poisoning in dogs. J Vet Pharmacol Ther 2008, 31, 143-149.

25. Yamato O., Hayashi M., Kasai E., Tajima M., Yamasaki M., Maede Y.: Reduced glutathione accelerates the oxidative damage produced by sodium n-propylthiosulfate, one of the causative agents of onion-induced hemolytic anemia in dogs. BBA-Gen Subjects 1999, 1427, 175-182.

26. Yanagita T., Han S.Y., Wang Y.M., Tsuruta Y., Anno T.: Cycloalliin, a cyclic sulfur imino acid, reduces serum triacylglycerol in rats. Nutrition 2003, 19, 140-143.

27. Zhai X., Chen X., Shi J., Shi D., Ye Z., Liu W., Li M., Wang Q., Kang Z., Bi H.: Lactulose ameliorates cerebral ischemiareperfusion injury in rats by inducing hydrogen by activating Nrf2 expression. Free Radic Biol Med 2013, 65, 731-741. 